\title{
Coherent Dedispersion: History and Results
}

\author{
Timothy H. Hankins ${ }^{1,2}$ \\ ${ }^{1}$ Physics Department, New Mexico Tech, Socorro, NM 87801 \\ ${ }^{2}$ National Radio Astronomy Observatory, Socorro, NM 87801 \\ email: thankins@aoc.nrao.edu
}

\begin{abstract}
The high time resolution afforded by coherent dedispersion has enabled precision pulsar timing, detailed studies of pulsar morphology, and has led to conclusions about the radio emission mechanism. The advance of technology in the last 50 years has enhanced the capability of coherent dedispersion, now used for most pulsar observing, by nearly six orders of magnitude. Although coherent dedispersion is now done mostly in software, in "earlier days" several novel hardware devices for real-time processing were developed.
\end{abstract}

Keywords. methods: data analysis, pulsars: general

\section{Introduction}

Dispersive propagation of pulsar signals through the ionized interstellar medium (ISM) smears temporal details as the signals sweep downward in frequency across a receiver passband. Fortunately there are several approaches to improving the time resolution, coherent dedispersion, incoherent dedispersion, hybrids of these two, and swept local oscillator techniques. The idea for coherent dedispersion was originated by Professor Kenneth Bowles at the University of California, San Diego. At an URSI Meeting in Washington in March, 1968, he heard the first reports of pulsar observations at Jodrell Bank and the Arecibo Observatory. He realized immediately that the ISM dispersion could be represented by an all-pass, phase changing filter, and that the resulting time smearing could be removed by passing the received signal through an inverse filter.

\section{Techniques}

Time resolution can be improved by appropriate delay of detected filter bank outputs (Hankins 1974) but the best time resolution that can be obtained by this method is when the filter bandwidth, $\Delta f$, is reduced until the dispersion sweep time across the band, $t_{\min }=|d f / d t|^{-1 / 2}$ is equal to the rise time of the filter, $1 / \Delta f$. For example, the best time resolution one can attain with this "incoherent dedispersion" or "post-detection delay compensation" for the millisecond pulsar B1937+214 at $400 \mathrm{MHz}$ is about $1 \%$ of the period, insufficient to resolve the short component on the trailing edge of the profile. For optimal time resolution one must use coherent dedispersion.

The formalism for coherent dedispersion is simple (Hankins 1971, Hankins \& Rickett 1975). In the following discussion time domain quantities are in lower case, frequency domain in upper case, the Fourier transform is indicated by $\Leftrightarrow$ and convolution by $\star$. We represent the signal emitted by the pulsar as $s(t) \Leftrightarrow S(f)$ and the transfer function of the ISM as $H(f)=e^{-i k(f) z} \Leftrightarrow h(t)$, where $z$ is the pulsar distance. Then the received signal is $s(t) \star h(t) \Leftrightarrow S(f) H(f)$. To recover $s(t)$ we multiply the Fourier transform of the received signal by $H^{-1}(f)$. Then we obtain $S(f) H(f) H^{-1}(f) \Leftrightarrow s(t)$. 


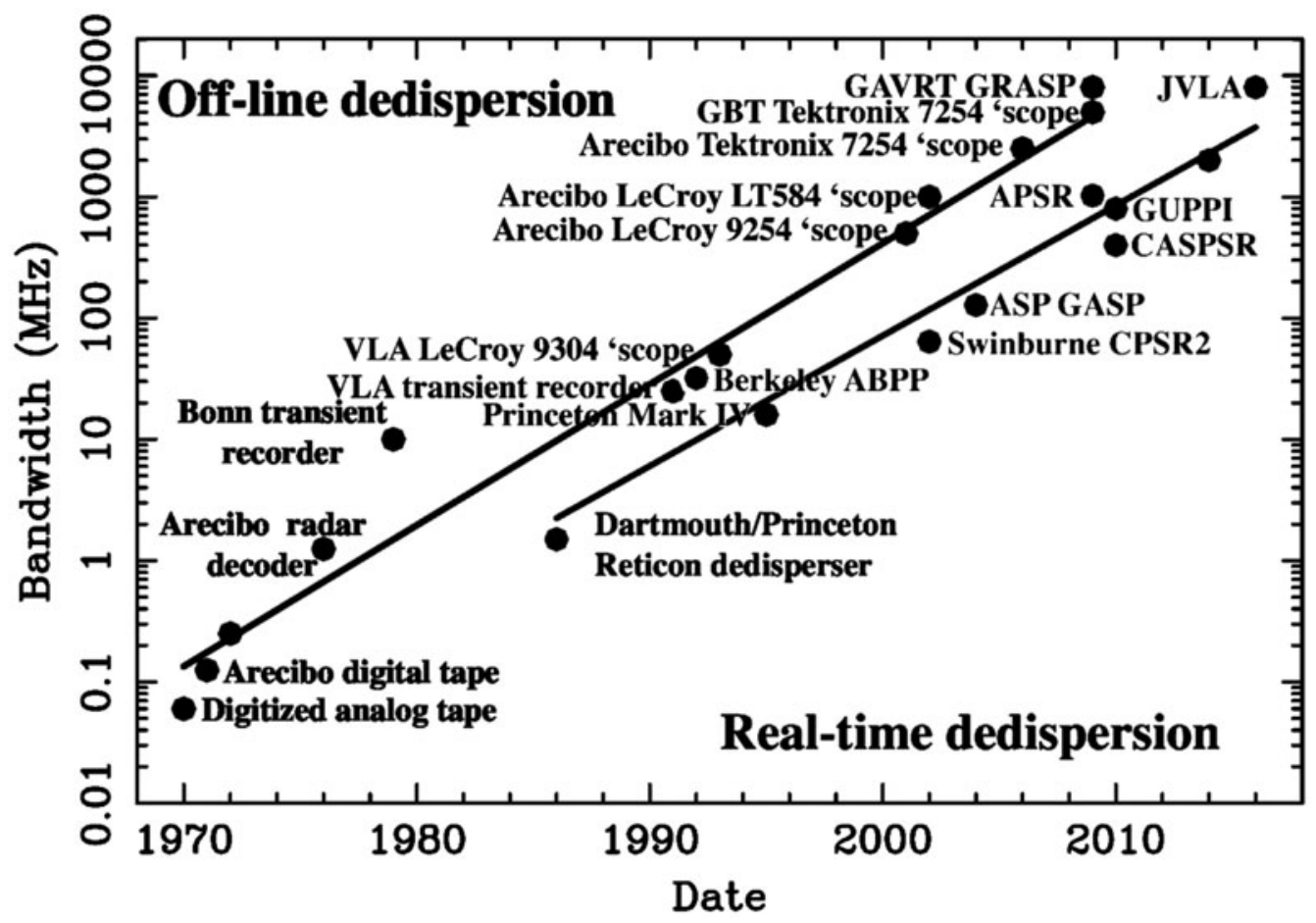

Figure 1. Moore's Law for coherent dedispersion

For my first attempt at coherent dedispersion in 1969 I recorded a $60 \mathrm{kHz}$ band of the received complex voltage on analog magnetic tape, then played back the tape at a slower rate to digitize it. Processing was done on a Sigma 5 computer with $48 \mathrm{kB}$ RAM and a $4 \mathrm{MB}$ disk drive. This worked, but it was crude. Subsequently I used the much faster digitizer at the Arecibo Observatory and found quasi-periodic intensity structure and short $(7 \mu \mathrm{s})$ micropulses from B0950+08 (Hankins 1971).

Realtime coherent dedispersion can be achieved in the time domain with a transversal filter. Of the several approaches (surface acoustic wave devices, tapped delay lines, etc.) I only know of two that were successful, the Dartmouth-Princeton Reticon Dedisperser (Hankins \& Rajkowski 1987), and part of the Berkeley Pulsar Processor (BPP). The former is based on the Reticon A5601 512-tap device designed for the chirp-z transform. The BPP instrument used a CMOS 1024-tap transversal filter (Backer et al. 1997).

An alternative configuration that has been very successful is a hybrid where coherent dedispersion is applied to each channel of a (usually digital) filter bank, implemented in software on computer clusters and graphical processing units. Hybrid dedispersers provide adequate resolution for many pursuits without the need for the extremely long Fourier transforms used for dedispersing a single very wide bandwidth.

Advances in technology have led to huge increases in the achievable dedispersed bandwidths. In Figure 1 I show the time line of improvement for some representative off-line dispersion removal (bandwidth doubling every 2.6 years), and hybrid systems (doubling time of 3 years). These systems have produced a long list of acronyms (APSR, BPP, CASPER, GUPPI, PUPPI, WAPP, etc.)

The upper limit of bandwidth is imposed on these instruments by the feed, receiver or intermediate frequency passband, currently about $2.5 \mathrm{GHz}$ at the Arecibo Telescope, 4 GHz at the Green Bank Telescope and $8 \mathrm{GHz}$ at the Jansky Very Large Array (JVLA). 

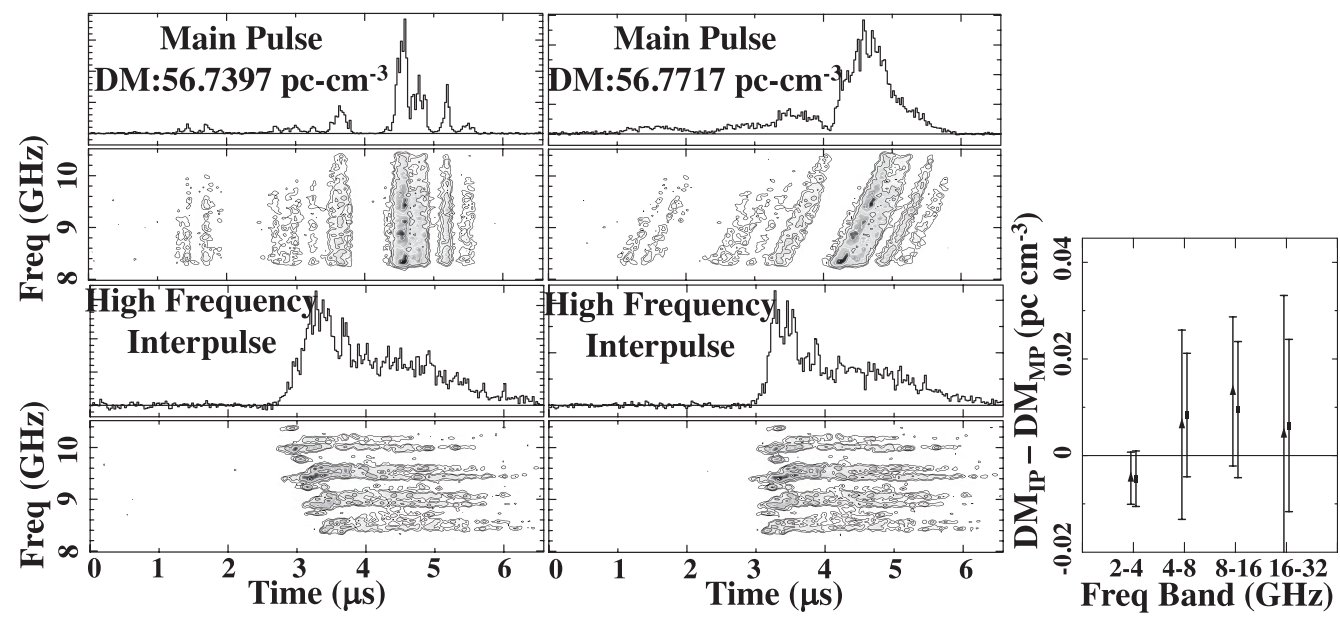

Figure 2. The left panels show the intensity and dynamic spectrum of a Main Pulse (upper panels) and High-Frequency Interpulse (lower panels) recorded about 12 minutes apart and processed identically. The center panels show the same pulses with the High-Frequency Interpulse processed with a larger DM to align the leading edges of the dynamic spectrum. The right panel shows the average dispersion measures for pulses recorded in four bands. The excess DM of the High-Frequency Interpulse may indicate a longer magnetospheric propagation path.

Glenn Jones, using a wideband feed developed by Sandy Weinreb for the Goldstone Apple Valley Radio Telescope, obtained a bandwidth of over $8 \mathrm{GHz}$ and has captured single pulses from the Crab from 2.5 to $10.5 \mathrm{GHz}$, proving that the Crab pulses are wideband (Hankins, Eilek \& Jones 2016).

\section{What coherent dedispersion has enabled}

Diagnostics for the emission mechanism. The nanosecond intensity structure predicted by Weatherall (1997) resulting from plasma turbulence in the pulsar magnetosphere was found using coherent dedispersion (Hankins et al. 2003).

Propagation. Precision determination of dispersion measure (DM) is used for ISM turbulence studies and measurements of possible possible radial proper motion of pulsars (Lam et al. 2016).

Precision timing. The accurate, high-definition average profiles afforded by coherent dedispersion are essential for gravitational wave, glitch and pulsar interior studies.

Discoveries. Although nebula echoes of the Crab pulsar radio signals were known from average profile work, the frequency dependence of very close-in echoes was found only by coherent dedispersion of the strong single pulses (Crossley et al. 2007).

The differences in DM between the single, bright Main Pulses and the High-Frequency Interpulses, shown in Figure 2, were determined using coherent dedispersion over a 2.5 $\mathrm{GHz}$ band (Hankins, Eilek \& Jones 2015). To measure the DM difference on these short pulses $(\approx 1-3 \mu \mathrm{s})$, coherent dedispersion was essential.

The Crab pulsar shows seven frequency dependent components in its average profile. For some time there was a discussion about the phase shift of $\approx 7^{\circ}$ of the interpulse between low $(\approx 1 \mathrm{GHz})$ and high $(\approx 10 \mathrm{GHz})$ frequencies. Recent observations at the JVLA (see Figure 3) have shown that the Low-Frequency Interpulse and High-Frequency Interpulse are clearly separate, distinct components. This is corroborated by the differences in emission statistics, polarization and frequency structure between these components (Hankins, Eilek \& Jones, 2015, 2016). 


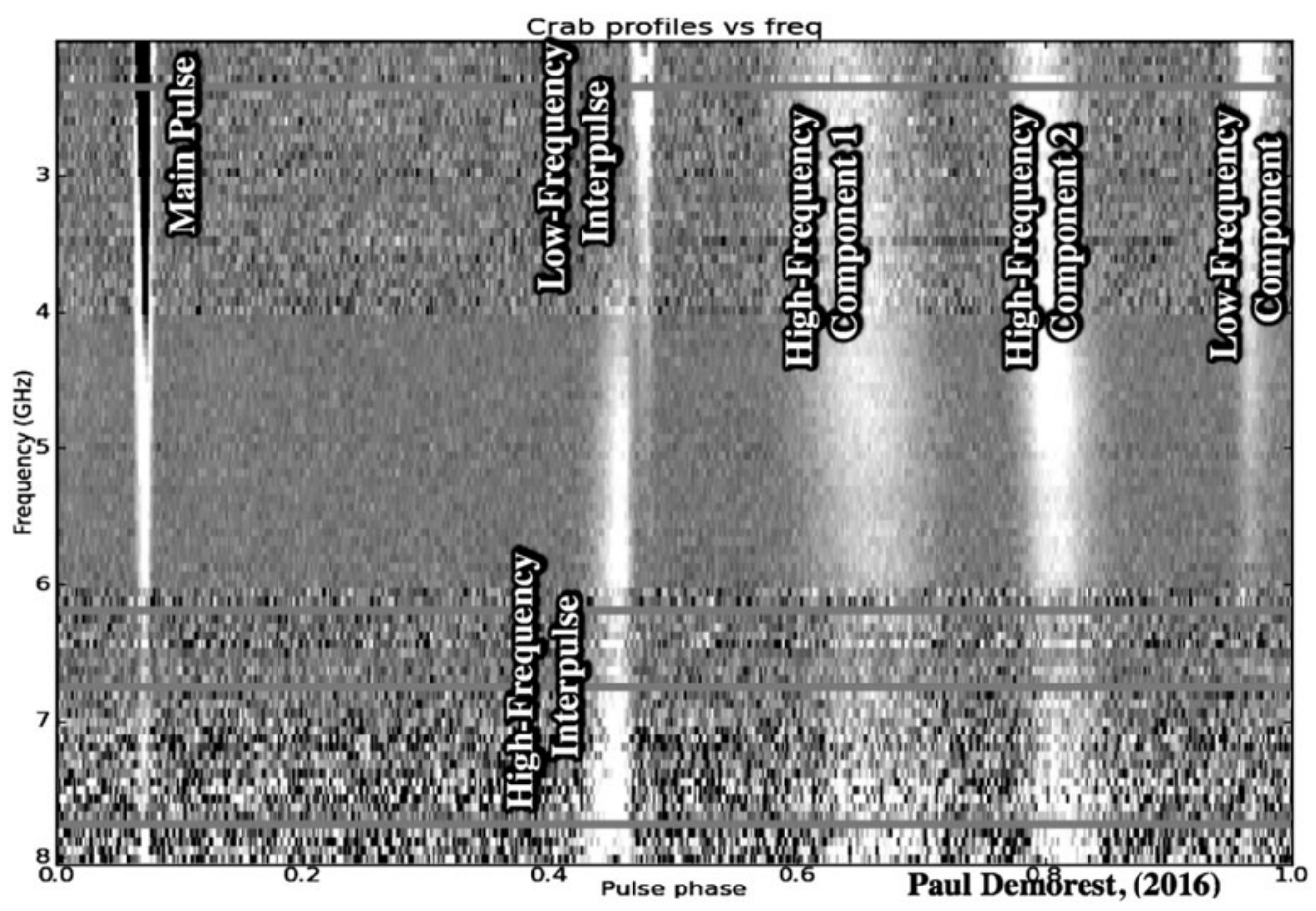

Figure 3. JVLA observations of the Crab average profile dynamic spectra obtained by Paul Demorest in three sessions from 2 to $8 \mathrm{GHz}$. The Low-Frequency Interpulse and High-Frequency Interpulse are clearly seen as distinct and independent emission components. The Low-Frequency Component is also shown, the origin of which has received very little attention.

\section{Future}

Coherent dedispersion will certainly continue to be used for observations in many areas of mainstream pulsar science. One can envision future pulsar searches where all processing for many simultaneous synthesized beams at single-dish telescopes or arrays will be done in real time. As technology continues to advance the speed of processing will improve and costs will decrease enabling even wider parameter space searching in dispersion, period and acceleration.

\section{References}

Backer, D. C., Dexter, M. R., Zepka, A., Ng, D., Werthimer, D. J., Ray, P. S., \& Foster, R. S. 1997, PASP, 109, 61

Crossley, J. H., Eilek, J. A., \& Hankins, T. H. 2007, ASPC, 365, 271

Hankins, T. H. 1971, ApJ, 169, 487

Hankins, T. H. 1974, $A \& \& A S, 15,363$

Hankins, T. H. \& Rajkowski, J. M. 1987, Rev. Sci. Inst., 58, 674

Hankins, T. H. \& Rickett, B. J. 1975, Meth. Comp. Phys., 14, (Academic, New York) p. 55

Hankins, T. H., Kern, J. S., Weatherall, J. C., \& Eilek, J. A. 2003, Nature, 422, 141

Hankins, T. H., Eilek, J. A., \& Jones, G. 2015, ApJ, 802, 130

Hankins, T. H., Eilek, J. A., \& Jones, G. 2016, ApJ, 833, 17

Lam, M. T., Cordes, J. M., Chatterjee, S., Jones, M. L., McLaughlin, M. A., \& Armstrong, J. W. 2016, ApJ, 821, 66

Weatherall, J. C. 1997, ApJ, 483, 402 